\title{
Path-integral quantum PATHTREE and PATHINT Algorithms
}

\author{
Lester Ingber \\ Lester Ingber Research \\ Ashland, Oregon 97520 \\ Email:ingber@alumni.caltech.edu [http://www.ingber.com] \\ Wed August 13 14:45:37 2016
}

\begin{abstract}
The author previously developed a generalization of a binomial tree algorithm, PATHTREE, to develop options pricing for multiplicative-noise models possessing quite generally time dependent and nonlinear means and variances. This code is generalized here for complex variable spaces, to produce qPATHTREE useful for quantum systems. As highlighted in this paper, a quantum version, qPATHTREE, has the ability to take into account time dependent modifications of an evolving system. qPATHTREE is shown to be useful to study some aspects of serial changes to systems. Similarly, another path-integral code, PATHINT, used for several previous systems is developed into qPATHINT. An example is given for a free particle, and it is explained why an n-tree generalization of qPATHTREE beyond the binomial tree is required for such systems, similar to code developed for qPATHINT. Potential applications in neuroscience and financial markets are discussed.
\end{abstract}

Index Terms: path integral; quantum mechanics; blockchains; parallel code; vector potential

\section{INTRODUCTION}

The author previously developed a generalization of a binomial tree algorithm, PATHTREE, to develop options pricing for multiplicative-noise models possessing quite generally time dependent and nonlinear means and variances [1]. Below, a short description is give of path integrals. In the next section, a brief review is given of PATHTREE [1], a fast and accurate C-coded algorithm evolves generally time-dependent short-time conditional probability distribution, in the context of path integrals, with quite general nonlinear means and variances, using a generalization of the limited binomial tree algorithm. This is followed by a brief description of a generalization of PATHTREE, qPATHTREE, a C code to evolve similar quantum mechanical functions in complex variable spaces. qPATHTREE is applied to a free particle. Calculations are give in the presence of random serial shocks to the particle. The current qPATHTREE is a binary tree. It is shown to be insufficient to handle oscillatory systems. The following section develops qPATHINT, another path-integral algorithm, which has been used for several systems, and which is capable of using an n-tree also useful for oscillatory systems $[2][3][4][5][6]$.

The example a free quantum particle was chosen to test treatment of its oscillatory properties. However, of course this system has an infinite domain, and so calculations performed here cannot be accurate given the finite ranges used. However, a proof of principle has been demonstrated, which is poised to handle quantum options on quantum systems, in finance and in blockchains. Quantum computing is here, and in the near future it will be applied to financial products, e.g., blockchains. It not very far-fetched to assume that soon there will be derivatives developed on these products. Then, as is the case in classical real spaces with PATHTREE and PATHINT, qPATHTREE and qPATHINT are now poised to calculate derivatives in quantum complex spaces. This is beyond simply using quantum computation of derivatives, since the space of the dependent variables themselves may live in quantum worlds [7][8][9][10][11][12]. The section following that describes a physical-biological problem that arose in neuroscience, in a project undertaken by the author, "Electroencephalographic field influence on calcium momentum waves." This project is the primary motivation to develop qPATHTREE. Several studies, fitting EEG data to a model of neocortex that explicitly accounts for multiple scales of neocortical interactions, Statistical Mechanics of Neocortical Interactions (SMNI) [13][14][15][16][2], were performed, wherein these $\mathrm{Ca}^{2+}$-wave effects modified parameterization of background SMNI parameters. qPATHTREE may be useful to determine how the regenerative process that defines $\mathrm{Ca}^{2+}$ waves also may produce reasonable shocks to the waves without seriously damaging its coherence properties. 
The conclusion stresses that these applications of qPATHTREE and qPATHINT give proofs of concept of these new algorithms and codes.

\subsection{Path Integrals}

The path-integral representation for $P$ for the short-time propagator is given in terms of a Lagrangian $\tilde{L}$ by

$$
\begin{gathered}
P\left(S^{\prime}, t^{\prime} \mid S, t\right)=\frac{1}{2 \pi g^{2} \Delta t} \exp (-\tilde{L} d t) \\
\tilde{L}=\frac{\left(\frac{d S}{d t}-f\right)^{2}}{2 g^{2}} \\
\frac{d S}{d t}=\frac{S^{\prime}-S}{d t}, d t=t^{\prime}-t
\end{gathered}
$$

The path integral defines the long-time evolution of $P$ in terms of the action $\tilde{A}$,

$$
\begin{gathered}
\tilde{P}[\tilde{S}(t)] d \tilde{S}(t)=\int \ldots \int \tilde{D} \tilde{S} \exp (-\tilde{A}) \\
\tilde{A}=\int_{t_{0}}^{t} d t \tilde{L}, \tilde{L}=\Lambda \Omega^{-1} \int d \vec{S} L \\
\tilde{D} \tilde{S}=\prod_{s=1}^{u+1} \prod_{v=1}^{\Lambda}\left(2 \pi d t g_{s}^{v 2}\right)^{-1 / 2} d S_{s}^{v} \delta\left[S_{t}=S(t)\right]\left[\delta\left[S_{0}=S\left(t_{0}\right)\right]\right.
\end{gathered}
$$

where $v$ labels the $\vec{S}$-space over the volume $\Omega$, and $s$ labels the $u+1$ time intervals, each of duration $d t$, spanning $\left(t-t_{0}\right)$. Note that the path integral is a faithful mathematical representation of (properly defined) Fokker-Planck partial differential equations and Langevin stochastic differential equations [17]. This paper explicitly uses the prepoint discretization.

\section{PATHTREE}

\subsection{Tree Algorithms}

Tree algorithms are generally derived from binomial random walks [18]. For many applications, these algorithms are more efficient for numerical computation, compared to their other mathematical representations as Langevin and Fokker-Planck equations [19][20]. These tree algorithms had previously typically been only well defined for specific functional forms of $f$ and $g$. The PATHINT algorithm deals with quite general $f$ and $g$ functions [4].

\subsection{BinOMIAL TREE}

In a two-step binomial tree, the step up $S u$ or step down $S d$ from a given node at $S$ is chosen to match the standard deviation of the differential process. The constraints on $u$ and $d$ are chosen as

$$
u d=1
$$

If probability $p$ is assigned to the up step $S u$, and $q=(1-p)$ to the down step $S d$, the matched mean and variance are

$$
\begin{gathered}
p S u+(1-p) S d=\langle S(t+\Delta t)> \\
S^{2}\left(p u^{2}+q d^{2}-(p u+q d)^{2}\right)=<(S(t+\Delta t)-<S(t+\Delta t)>)^{2}>
\end{gathered}
$$

\subsubsection{DEFICIENCY OF STANDARD ALgORITHM TO ORDER $\sqrt{d t}$}

The 4 unknowns $\{u, d, p, q\}$ are calculated by imposing the normalization of the probability and matching the first two moments conditioned by the value $S$ at $t-\Delta t$, using the variance of the exact probability distribution $P\left(S, t \mid S_{0}, t_{0}\right)$. One additional condition is arbitrary and is usually used to symmetrize the tree, e.g., $u d=1$.

The above procedure cannot be applied to a general nonlinear diffusion process, as previous knowledge is required of terms of $O(\Delta t)$ in the formulas of quantities $\{u, p\}$ obtained from a finite time expansion of the exact solution $P$ sought. 
The discrete numerical approximation obtained does not converge to the proper solution.

\subsection{PATHTREE}

A general path-integral solution of the Fokker-Plank equation, including the well-known Black-Scholes equation [21], can be numerically calculated using the PATHINT algorithm. Although this approach leads to very accurate results, it is computationally intensive. To $O(\Delta t)$, in the prepoint discretization relevant to the construction of a tree,

$$
\begin{gathered}
P\left(S^{\prime}, t^{\prime} \mid S, t\right)=\frac{1}{\sqrt{2 \pi \Delta t g^{2}}} \exp \left(-\frac{\left(S^{\prime}-S-f d t\right)^{2}}{2 g^{2} \Delta t}\right) \\
\Delta t=t^{\prime}-t
\end{gathered}
$$

Valid for displacements $S^{\prime}$ from $S$ "reasonable" as measured by the standard deviation $g \sqrt{\Delta t}$, which is the basis for the construction of meshes in the PATHINT algorithm. PATHTREE starts with the initial value $S_{0}$, with successive increments

$$
\begin{gathered}
S_{i+1}=S_{i}+g \sqrt{\Delta t}, S_{i}>S_{0} \\
S_{i-1}=S_{i}-g \sqrt{\Delta t}, S_{i}<S_{0}
\end{gathered}
$$

Where $g$ is evaluated at $S_{i}$. Up and down probabilities $p$ and $q$, resp., are defined in an abbreviated notation, as

$$
\begin{gathered}
p=\frac{P(i+1 \mid i ; \Delta t)}{P(i+1 \mid i ; \Delta t)+P(i-1 \mid i ; \Delta t)} \\
q=1-p
\end{gathered}
$$

Where the short-time transition probability densities $P$ 's are calculated from Eq. (). Like PATHINT, PATHTREE easily handles American early-exercise, time-dependent and variable-dependent dividends, volatility, cost of carry and interest rates, etc. PATHTREE also can be used to fit shape-dependent conditional probability densities to near real-time strike data, e.g., a "bottom-up" approach to "smiles" (patterns of implied volatility). The same should be expected of qPATHTREE in complex-variable spaces.

\subsection{Direct Calculation of Probability}

The formula developed for PATHTREE [1], is a discretized version of the Chapman-Kolmogorov equation

$$
p\left(x_{j}, t_{i}\right)=p\left(x_{j-1}, t_{i-1}\right) p u_{j-1}+p\left(x_{j+1}, t_{i-1}\right) p d_{j+1}
$$

After computing the absolute probabilities at the final nodes, a proper estimation of the density is obtained by scaling the probabilities by the average of sizes of the two adjacent intervals, density ${ }_{i}=p_{i} /\left(\left(S_{i+2}-S_{i-2}\right) / 2\right)$.

\subsection{QPATHTREE}

The PATHTREE C code comprises about 7000 lines of code. The GCC code was rewritten to use complex double variables instead of double variables. The code also runs under CLANG. A lot of changes were made to $\mathrm{I} / \mathrm{O}$, since this requires separation of real and imaginary parts of each number. This code utilizes parallel processing on NSF.gov XSEDE.org platforms. OpenMP is used for parallel processing of some loops within each run. MPI is used for parallel processing of multiple runs. The section dealing with the evolution of the probability distribution was modified. The Lagrangian argument of the probability distribution is written in complex form. Similar to PATHTREE, in qPATHTREE, for systems that define complex variables over real-variable spaces, the real part of complex value of the square-root of the diffusion is used to generate the mesh of the tree.

\section{PATHINT}

This approach was motivated by a previous non-Monte-Carlo multivariable generalization of a numerical path-integral algorithm [22][23][24], PATHINT, used to develop the long-time evolution of the short-time probability distribution as used in several studies in chaotic systems [3][6], neuroscience [2][3][5], and financial markets, including two-variable volatility of volatility [4]. These studies suggested applications of some aspects of this algorithm to the standard binomial tree. PATHINT develops bins $T_{i j}$ about diagonal terms in the Lagrangian.

$$
P_{i}(t+\Delta t)=T_{i j}(\Delta t) P_{j}(t)
$$




$$
T_{i j}(\Delta t)=\frac{2}{\Delta S_{i-1}+\Delta S_{i}} \int_{S_{i}-\Delta S_{i-1} / 2}^{S_{i}+\Delta S_{i} / 2} d S \int_{S_{j}-\Delta S_{j-1} / 2}^{S_{j}+\Delta S_{j} / 2} d S^{\prime} G\left(S, S^{\prime} ; \Delta t\right) .
$$

$T_{i j}$ is a banded matrix representing the Gaussian nature of the short-time probability centered about the (varying) drift. Fitting data with the short-time probability distribution, effectively using an integral over this epoch, permits the use of coarser meshes than the corresponding stochastic differential equation. The coarser resolution is appropriate, typically required, for numerical solution of the time-dependent path-integral: By considering the contributions to the first and second moments of $\Delta S^{G}$ for small time slices $\theta$, conditions on the time and variable meshes can be derived [22].

The time slice essentially is determined by $\theta \leq \bar{L}^{-1}$, where $\bar{L}$ is the "static" Lagrangian with $d S^{G} / d t=0$, throughout the ranges of $S^{G}$ giving the most important contributions to the probability distribution $P$. The variable mesh, a function of $S^{G}$, is optimally chosen such that $\Delta S^{G}$ is measured by the covariance $g^{G G^{\prime}}$, or $\Delta S^{G} \approx\left(g^{G G} \theta\right)^{1 / 2}$. PATHINT was generalized to process arbitrary $\mathrm{N}$ variable spaces, but in practice only $\mathrm{N}=2$ was used because of intensive computer resources.

The author was Principal Investigator, National Science Foundation (NSF) Pittsburgh Supercomputing Center (PSC) Grant DMS940009P during 1994-1995 on a project, "Porting Adaptive Simulated Annealing and Path Integral Calculations to the Cray; Parallelizing ASA and PATHINT Project (PAPP)". Eight volunteers were selected from many applicants and the PATHINT code was seeded to work on parallel machines. No further work has been done since that time to further develop parallel-coded applications.

\subsection{QPATHINT}

Similar to the development of qPATHTREE described above, the PATHTREE C code of about 7500 lines of code was rewritten for GCC code to use complex double variables instead of double variables.

\subsection{QPATHTREE}

\section{FREE PARTICLE EXAMPLE}

A simple example to test qPATHTREE is the evolution of the propagator $P$ for a free particle,

$$
P\left(x^{\prime}, t^{\prime} ; x, t\right)=<\psi\left(x^{\prime}, t^{\prime}\right) \mid \psi(x, t)>=\left(2 \pi i \hbar\left(t^{\prime}-t\right) / m\right)^{-1 / 2} \exp \left(i m \frac{\left(x^{\prime}-x\right)^{2}}{2 \hbar\left(t^{\prime}-t\right)}\right)
$$

The long-time solution has the same form, with $t=t_{0}$ and $t^{\prime}=t_{f}$. For brevity, take $m=1$ and $\hbar=1 . P$ is a complex-variable function over a real-variable space $x$. As noted above, the $x$-mesh for qPATHTREE is chosen to be the real part of $(i \hbar m)^{1 / 2}$. The number of iterations in the tree were initially determined by $\left(t_{f}-t_{0}\right) / 10^{-2}$, but larger numbers of iterations only increases the number of oscillations, and the fit in the middle sections remains the same. Therefore, this number was chosen to be $\min \left[200,\left(t_{f}-t_{0}\right) / 10^{-2}\right]$. Fig. 1 gives graphical results for qPATHTREE calculations superimposed on the exact solutions, for times corresponding to $t_{f}-t_{0}$ equal to $0.5,2$ and 20 .

\subsubsection{Serial SHOCKS}

In Figs. 2 and 3, calculations are give in the presence of random serial shocks to the particle. The standard C-code uniform integer random number generator, rand(), was scaled to develop random real numbers within [-1,1], which were multiplied by next value of the dependent variable times a scale factor. Shapes of the distribution are generally retained for all shocks relative to no shocks with parameter 0 , with more disruptions at higher shocks and longer propagation. Higher shocks gave severe large disturbances at negative $x$, so the $P$-range was cut off at \pm 1 . Note that the qPATHTREE imaginary part of $P$ agrees with the exact imaginary part at the center point. 
International Journal of Innovative Research in Information Security (IJIRIS) ISSN: 2349-7017(0) Issue 05, Volume 3 (August 2016)

ISSN: 2349-7009(P)

www.ijiris.com

$[\mathrm{B}] 0.4 \mathrm{TW}$

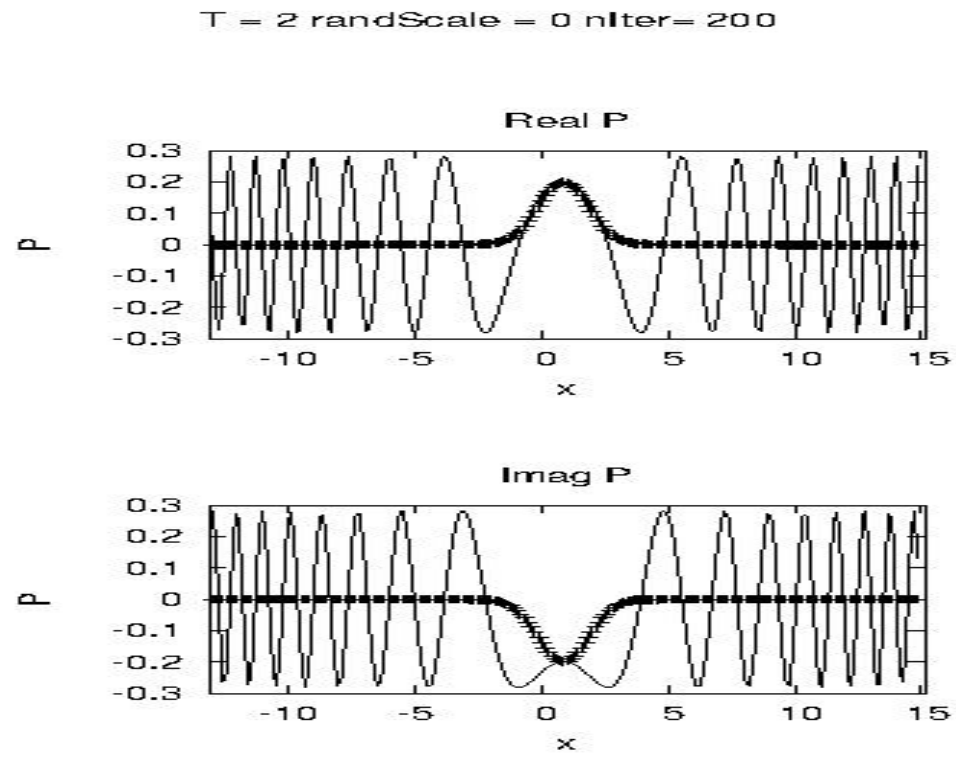

Figure 1: time $=2$

[B]0.4TW

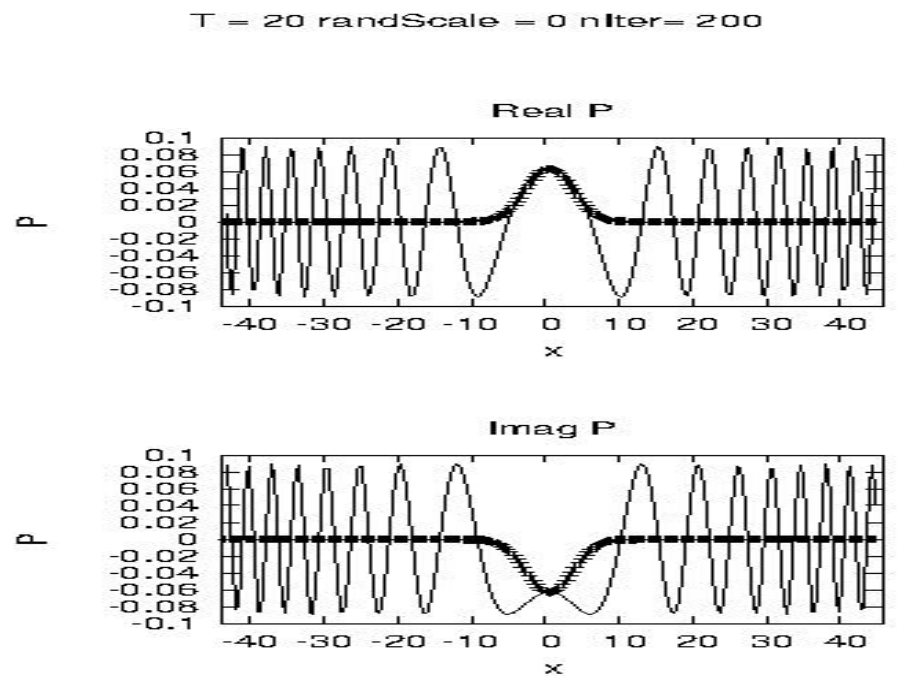

Figure 2: time $=20$

Figure 3: Real and Imaginary parts of a free particle propagator evolved with qPATHREE are shown over periods of (a) 2 and (b) 20, with a magnitude of shock set to 0. qPATHTREE calculations are shown as points. The exact analytical solutions are shown as lines. 
International Journal of Innovative Research in Information Security (IJIRIS) ISSN: 2349-7017(0) Issue 05, Volume 3 (August 2016)

ISSN: 2349-7009(P)

www.ijiris.com

$[B] 0.4 \mathrm{TW}$

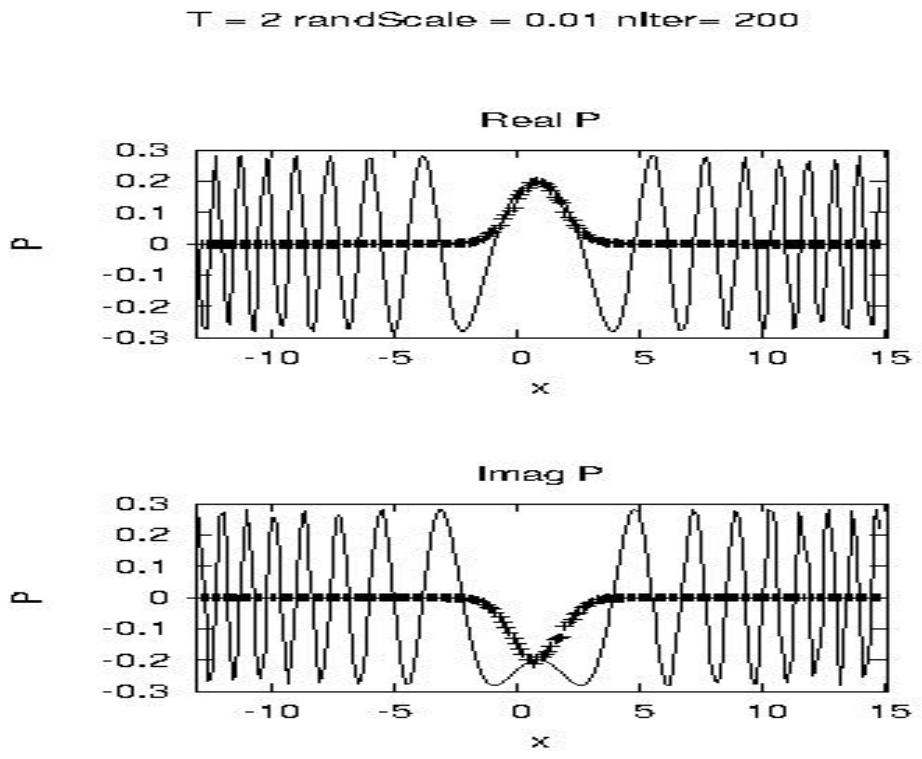

Figure 4: time $=2$

[B]0.4TW

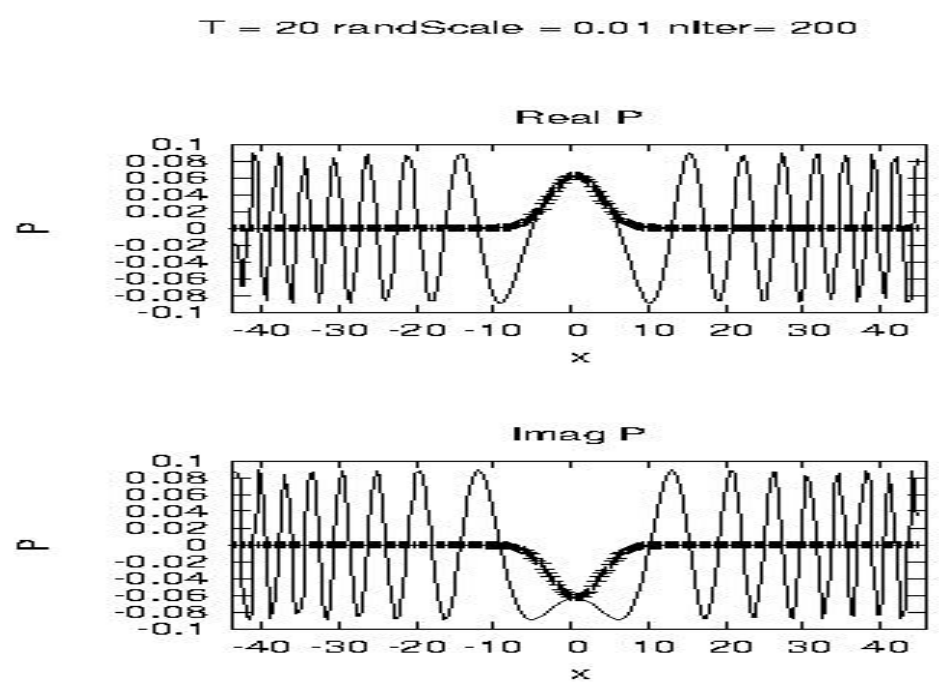

Figure 5: time $=20$

Figure 6: Real and Imaginary parts of a free particle propagator evolved with qPATHREE are shown over periods of (a) 2 and (b) 20, with a magnitude of shock set to 0.01. qPATHTREE calculations are shown as points. The exact analytical solutions are shown as lines. 
International Journal of Innovative Research in Information Security (IJIRIS) ISSN: 2349-7017(0) Issue 05, Volume 3 (August 2016)

ISSN: 2349-7009(P)

www.ijiris.com

$[\mathrm{B}] \mathbf{0 . 4 T W}$

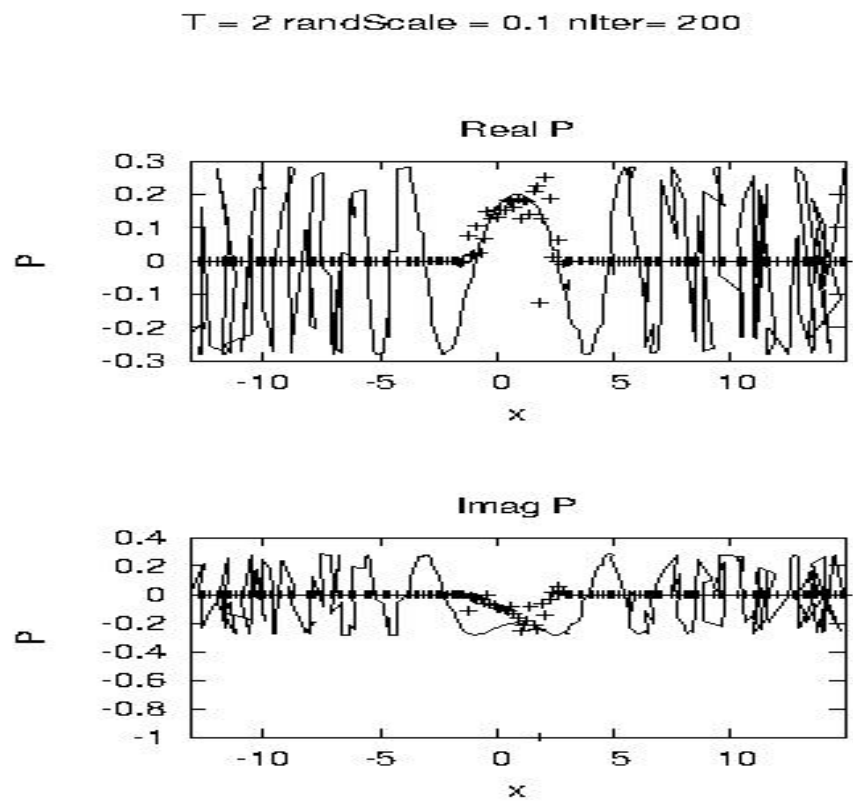

Figure $7:$ time $=2$

[B]0.4TW

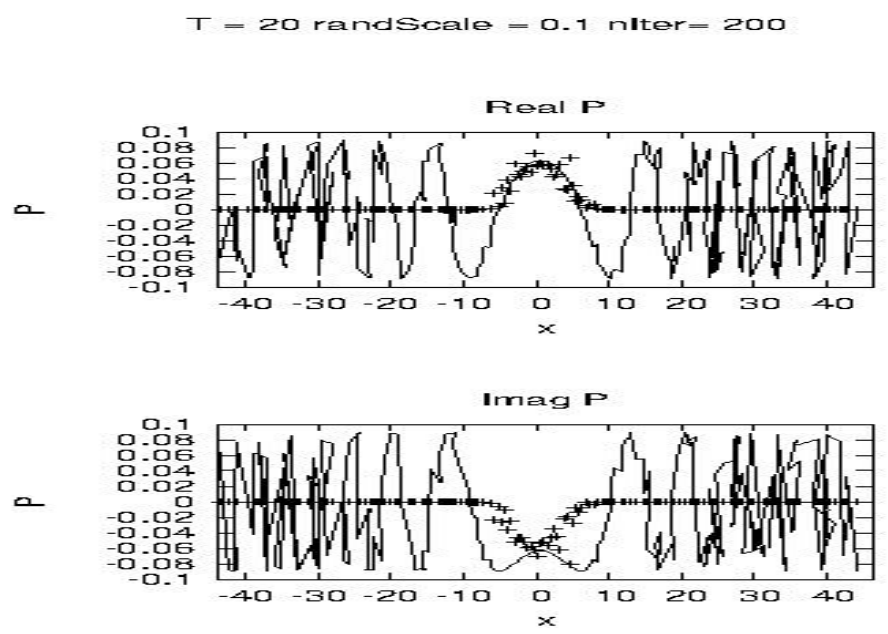

Figure 8: time $=20$

Figure 9: Real and Imaginary parts of a free particle propagator evolved with qPATHREE are shown over periods of (a) 2 and (b) 20, with a magnitude of shock set to 0.1. qPATHTREE calculations are shown as points. The exact analytical solutions are shown as lines. 


\subsubsection{QPATHTREE FLAWS}

Note that qPATHTREE, as reported here, simply uses this binary tree to develop a wave function, not the probability of the square of the evolving wave function. However, even at this stage, there is a flaw in the current qPATHTREE code: The oscillatory behavior of the analytic solution is not replicated. However, in the region of the main peak, qPATHTREE agrees very well with the analytic solution. This particular test system has zero drift and constant diffusion, so that every node in the tree just tests the same $d x$ mesh, with no opportunity to explore any other $x$ dependence. It is clear that just using a binomial tree for this system is insufficient to capture functional behavior just one standard deviation on each side of a branch. Rather, an n-tree is required with at least several points of deviations on each side of the each branch.

\subsection{QPATHINT}

PATHINT, and the qPATHINT currently under development, already is coded to span each branch for any number of offsets from the $d x$ diagonal read in as a parameter to the code. qPATHINT as reported here, normalizes the evolving matrix of distributions according the the square of the evolving wave function $\left(\psi \psi^{*}\right)$.

\subsubsection{Propagation Of IMPUlSE With Serial ShOcks}

An example of the use of qPATHINT is to study the propagation of an initial impulse to the free quantum particle at $t=0$ and $x=0$. For this example, this initial propagator gives the same results after 100 time slices as using the free propagator itself for the initial condition. Fig. 4 gives graphical results for qPATHINT calculations; normalization at each time slice is being investigated. Real and Imaginary parts of a free particle propagator evolved with qPATHINT are shown over a periods 0.5 , with 282 points of $x$-mesh in the path for 100 time-slices, for an off-diagonal offset of 1 . Similar to the tests with qPATHTREE, shocks are given as multiple of variables during each time-slice as 0, 0.01 and 0.1 . Note the absence of oscillatory behavior. Fig. 5 gives graphical results for qPATHINT calculations for an off-diagonal offset of 3 (on each side of the diagonal). Shocks are given as multiple of variables during each time-slice as $0,0.01$ and 0.1 . Note that oscillatory behavior captured using information further from the diagonal. With extreme shocks at 0.1 , the $x$ variables are randomly displaced each time slice (as described above for qPATHTREE), causing cancellations of the oscillations.

[в]0.3TW
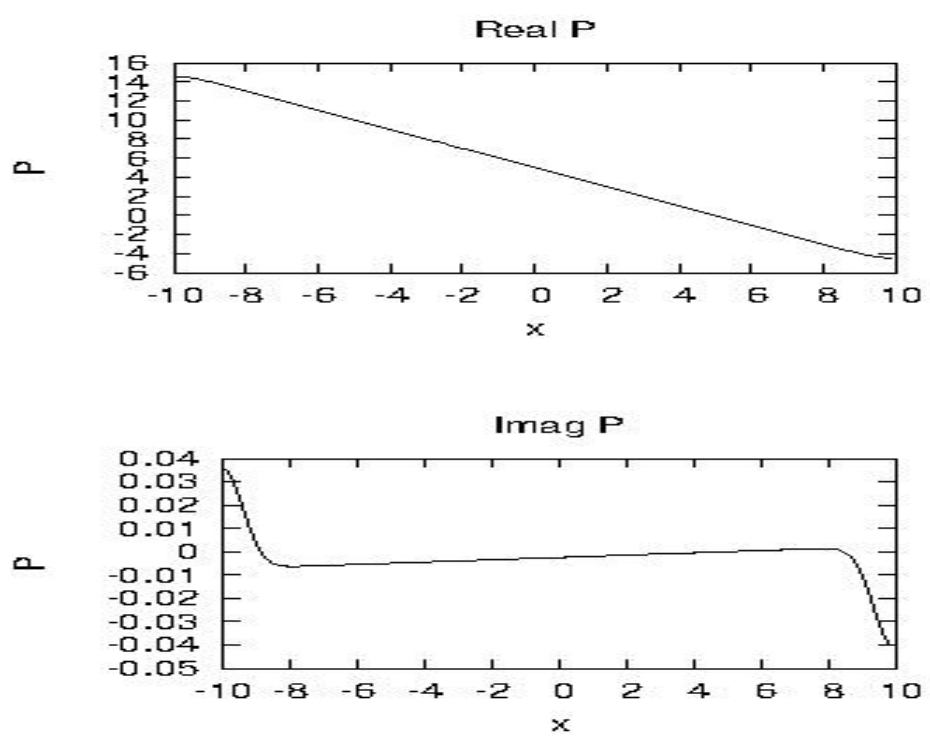

Figure 10: shock $=0$ 
International Journal of Innovative Research in Information Security (IJIRIS) ISSN: 2349-7017(0)
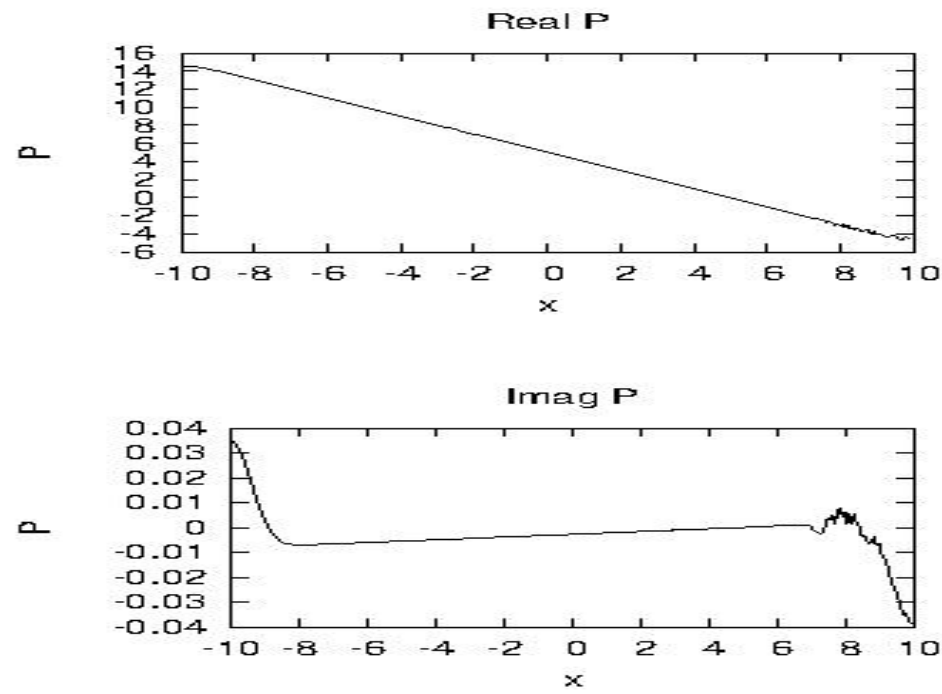

Figure 11: shock $=0.01$

[B]0.3TW
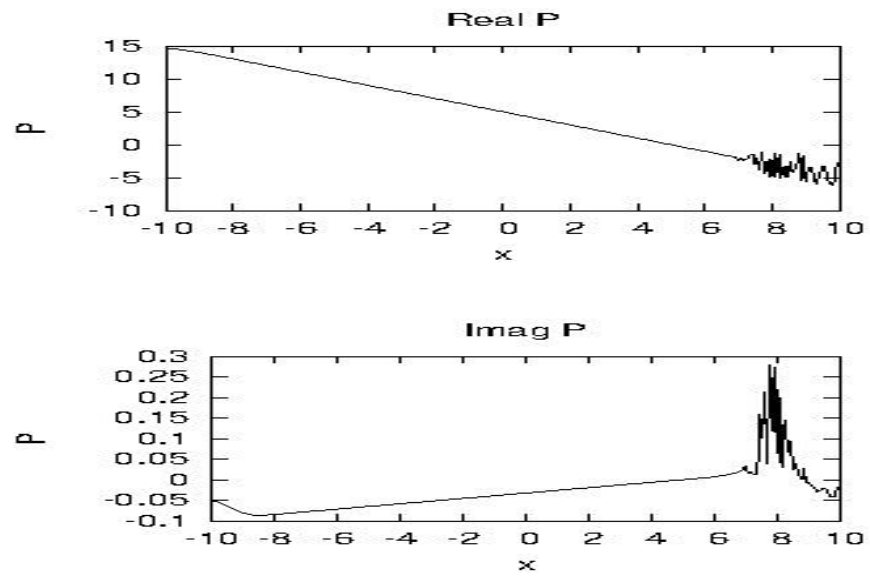

Figure 12: shock $=0.1$

Figure 13: Real and Imaginary parts of a free particle propagator evolved with qPATHINT are shown over a periods 0.5 with 100 intermediate time points in the path, for an off-diagonal offset of 1 . Shocks are 0 in (a), 0.01 in (b), and 0.1 in (c). 
International Journal of Innovative Research in Information Security (IJIRIS) ISSN: 2349-7017(0) Issue 05, Volume 3 (August 2016)

ISSN: 2349-7009(P)

www.ijiris.com

[B]0.3TW
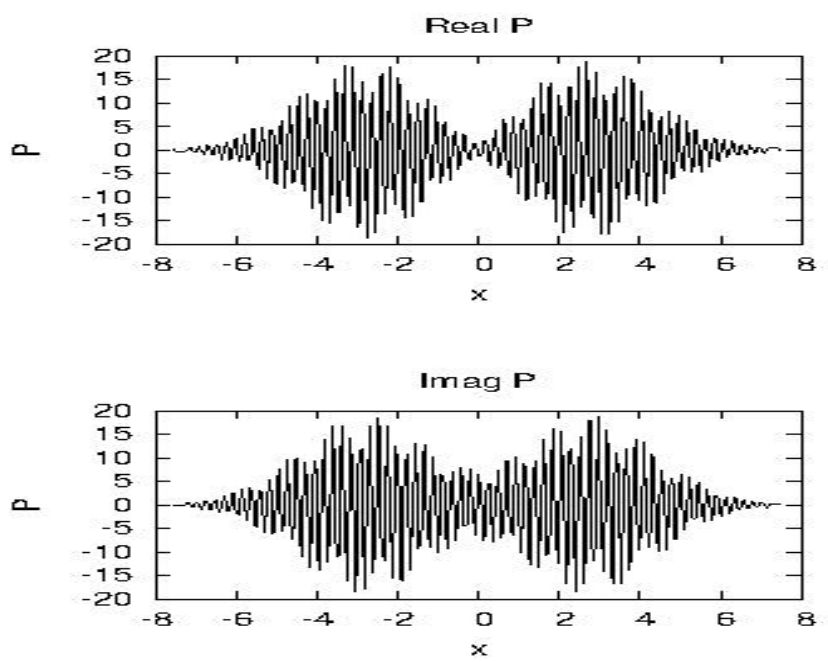

Figure 14: shock $=0$

[B]0.3TW
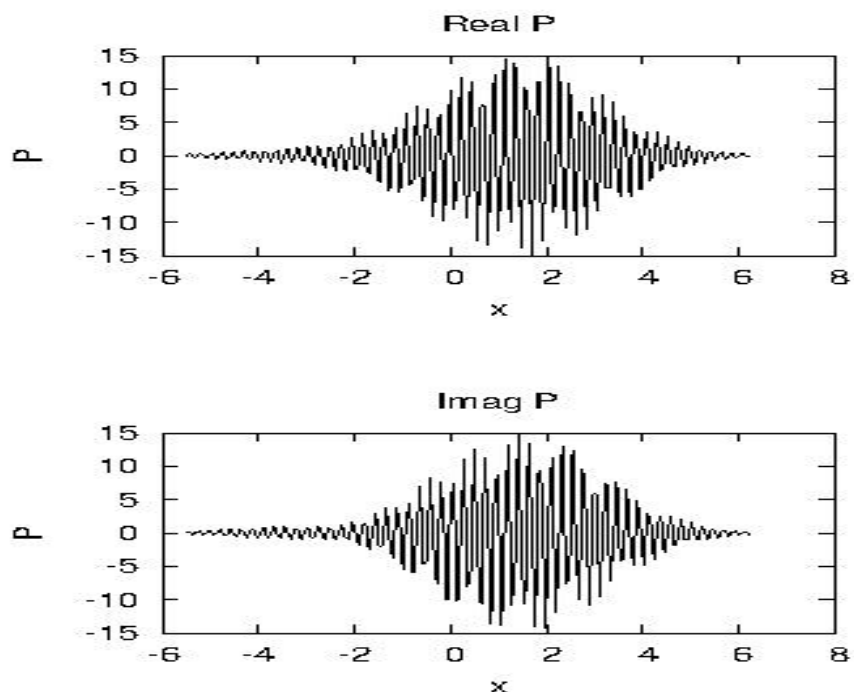

Figure 15: shock $=0.01$ 
$[\mathrm{B}] 0.3 \mathrm{TW}$
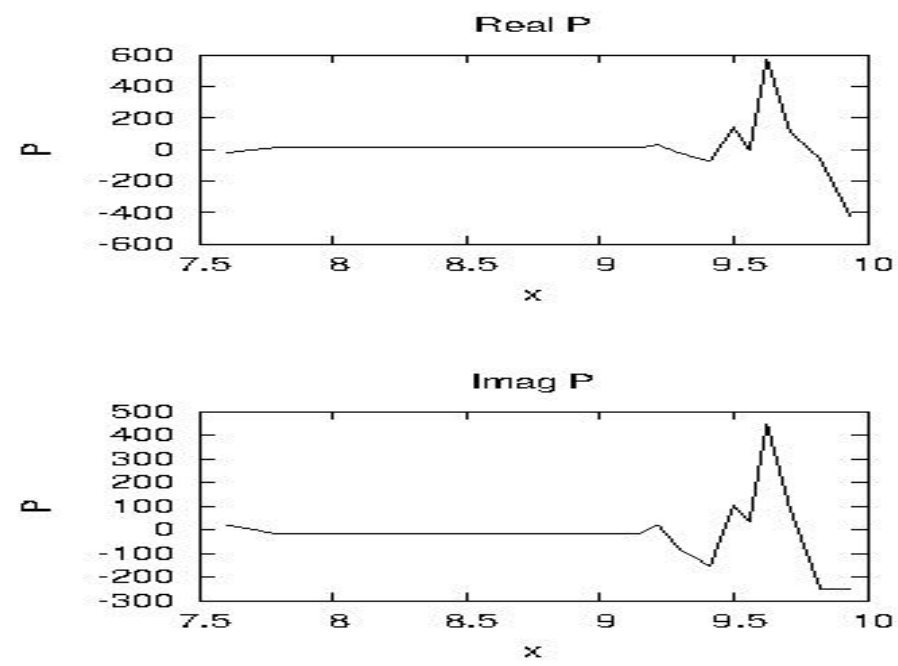

Figure 16: shock $=0.1$

Figure 17: Real and Imaginary parts of a free particle propagator evolved with qPATHINT are shown over a periods 0.5 with 100 intermediate time points in the path, for an off-diagonal offset of 3. Shocks are 0 in (a), 0.01 in (b), and 0.1 in (c).

\section{EEG INFLUENCE on CALCIUM MOMENTUM WAVES}

This section gives a summary of the XSEDE project that was developed between February 2013 through June 2016 , "Electroencephalographic field influence on calcium momentum waves", which generated interest in developing qPATHTREE and qPATHINT from PATHTREE and PATHINT, resp. This is just one example of studies that may take advantage of these new non-Monte-Carlo algorithms to study sequential development of quantum systems. In addition to the intrinsic interest of researching STM and multiple scales of neocortical interactions via EEG data, there is interest in researching possible quantum influences on highly synchronous neuronal firings relevant to STM to understand possible connections to "free will" [25][26].

\subsection{Molecular Processes Contributing to Synaptic Interactions}

There are many studies on tripartite neuron-astrocyte interactions [27], and on $\mathrm{Ca}^{2+}$ waves at tripartite sites. The short summary below is presented to set the context for SMNI calculations of probability distributions of synaptic activity that include background contributions. Several studies have shown that glutamate release from astrocytes through a $\mathrm{Ca}^{2+}$-dependent mechanism can activate receptors located at the presynaptic terminals. Regenerative intercellular calcium waves (ICWs) can travel over 100s of astrocytes, encompassing many neuronal synapses. These ICWs are documented in the control of synaptic activity [28]. Analysis of fluorescence accumulation clearly demonstrates that glutamate is released in a regenerative manner, with subsequent cells that are involved in the calcium wave releasing additional glutamate [29].

Although the full set of mechanisms affecting $\left[\mathrm{Ca}^{2+}\right]$ and the influences of $\mathrm{Ca}^{2+}$ on other mechanism are not yet fully understood and experimentally verified, it is clear that $\mathrm{Ca}^{2+}$ waves exist in intercellular and in intracellular media [30]. There are regenerative as well as non-regenerative processes observed, both "locally" at cellular sites as well as into "expanded" regions through which $\mathrm{Ca}^{2+}$ travel at relatively fast velocities for large distances over relatively long periods of time [31][32][33]. $\mathrm{Ca}^{2+}$ affects spontaneous synaptic production of glutamate. 


\subsection{INFLUENCE OF SYNCHRONOUS NeURAL FiringS}

Results of SMNI fits to EEG data gave strong confirmation of the SMNI model of short-term memory (STM), and give support to a basic physical mechanism that couples highly synchronous firings to control underlying molecular processes, the canonical momentum $\mathrm{P}, \mathrm{P}=\mathbf{p}+q \mathbf{A}$, where $\mathbf{p}$ is the momenta of a $\mathrm{Ca}^{2+}$ wave, $q$ the charge of $\mathrm{Ca}^{2+}$, $q=-2 e, e$ the magnitude of the charge of an electron [32]. Previous papers have used classical physics to calculate and compare the molecular $\mathbf{p}$ and large-scale q $\mathbf{A}$ components of $\mathrm{P}$, demonstrating that indeed they of comparable magnitudes [34][35][33][36]. Also, in the context of quantum mechanics, the wave function of the $\mathrm{Ca}^{2+}$-wave system was calculated, and it was demonstrated that overlap with multiple collisions, due to their regenerative processes, during the observed long durations of typical $\mathrm{Ca}^{2+}$ waves [31][33] support a Zeno effect [37][38][39][40][41][42][43][44] promoting long coherence times.

\subsubsection{Classical Physics of P}

Previous papers have modeled minicolumns as wires which support neuronal firings, due largely from large neocortical excitatory pyramidal cells in layer V (of six), giving rise to currents which give rise to electric potentials measured as scalp EEG [34][35][36]. This gives rise to a magnetic vector potential

$$
\mathbf{A}=\frac{\mu}{4 \pi} \mathbf{I} \log \left(\frac{r}{r_{0}}\right)
$$

Which has an insensitive $\log$ dependence on distance. In the brain, $\mu \approx \mu_{0}$, where $\mu_{0}$ is the magnetic permeability in vacuum $=4 \pi 10^{-7} \mathrm{H} / \mathrm{m}$ (Henry/meter), where Henry has units of $\mathrm{kg}-\mathrm{m}-\mathrm{C}^{-2}$, which is the conversion factor from electrical to mechanical variables. For oscillatory waves, the magnetic field $\mathbf{B}=\nabla \times \mathbf{A}$ and the electric field $\mathbf{E}=\frac{i c}{\omega} \nabla \times \nabla \times \mathbf{A}$ do not have this $\log$ dependence on distance. The magnitude of the current is taken from experimental data on dipole moments $\mathbf{Q}=|\mathbf{I}| z$ where $\hat{\mathbf{z}}$ is the direction of the current $\mathbf{I}$ with the dipole spread over $z$. $\mathbf{Q}$ ranges from $1 \mathrm{pA}-\mathrm{m}=10^{-12}$ A-m for a pyramidal neuron [45], to $10^{-9}$ A-m for larger neocortical mass [46]. These currents give rise to $q \mathbf{A}$ on the order of $10^{-28} \mathrm{~kg}-\mathrm{m} / \mathrm{s}$. $\mathbf{p}$ from one $\mathrm{Ca}^{2+}$ ion in a wave is typically on the order of $10^{-30} \mathrm{~kg}-\mathrm{m} / \mathrm{s}$, and this can be multiplied by the number of ions in a wave, e.g., 100's to 1000 's.

\subsubsection{QUantum Physics of P}

Previous papers have detailed quantum calculations of the wave function of $\mathrm{Ca}^{2+}$ waves in the presence of $\mathbf{A}$ [31][33]. The normalized wave function in momentum space, $\phi(\mathbf{p}, t)$, is

$$
\begin{gathered}
\phi(\mathbf{p}, 0)=\left(2 \pi(\Delta \mathbf{p})^{2}\right)^{-3 / 4} e^{-\left(\mathbf{p}-\mathbf{p}_{0}\right)^{2} /\left(4(\Delta \mathbf{p})^{2}\right)} \\
U(\mathbf{p}, t)=e^{\left.-i(\mathbf{p}+q \mathbf{A})^{2} t\right) /(2 m \hbar)} \\
\phi(\mathbf{p}, t)=\phi(\mathbf{p}, 0) U(\mathbf{p}, t)
\end{gathered}
$$

In momentum space the wave packet, consider $\phi(\mathbf{p}, t)$ being "kicked" from $\mathbf{p}$ to $\mathbf{p}+\delta \mathbf{p}$, and simply assume that random repeated kicks of $\delta \mathbf{p}$ result in $\langle\delta \mathbf{p}\rangle \approx 0$, and each kick keeps the variance $\Delta(\mathbf{p}+\delta \mathbf{p})^{2} \approx \Delta(\mathbf{p})^{2}$. Then, the overlap integral at the moment $t$ of a typical kick between the new and old state is

$$
\begin{gathered}
\left\langle\phi^{*}(\mathbf{p}+\delta \mathbf{p}, t) \mid \phi(\mathbf{p}, t)\right\rangle=e^{\frac{i \kappa+\rho}{\sigma}} \\
\kappa=8 \delta \mathbf{p} \Delta \mathbf{p}^{2} \hbar m\left(q \mathbf{A}+\mathbf{p}_{0}\right) t-4\left(\delta \mathbf{p} \Delta \mathbf{p}^{2} t\right)^{2} \\
\rho=-(\delta \mathbf{p} \hbar m)^{2} \\
\sigma=8(\Delta \mathbf{p} \hbar m)^{2}
\end{gathered}
$$

Where $\phi(\mathbf{p}+\delta \mathbf{p}, t)$ is the normalized wave function in $\mathbf{p}+\delta \mathbf{p}$ momentum space. A crude estimate is obtained of the survival time $A(t)$ and survival probability $p(t)$ [38],

$$
\begin{gathered}
A(t)=\left\langle\phi^{*}(\mathbf{p}+\delta \mathbf{p}, t)\right| \phi(\mathbf{p}, t)> \\
p(t)=|A(t)|^{2}
\end{gathered}
$$


These numbers yield:

$$
<\phi^{*}(\mathbf{p}+\delta \mathbf{p}, t) \mid \phi(\mathbf{p}, t)>=e^{i\left(1.67 \times 10^{-1} t-1.15 \times 10^{-2} t^{2}\right)-1.25 \times 10^{-7}}
$$

Even many small repeated kicks do not appreciably affect the real part of $\phi$, and these projections do not appreciably destroy the original wave packet, giving a survival probability per kick as $p(t) \approx \exp \left(-2.5 \times 10^{-7}\right) \approx 1-2.5 \times 10^{-7}$. Both time-dependent phase terms in the exponent are sensitive to time scales on the order of $1 / 10 \mathrm{~s}$, scales prominent in STM and in synchronous neural firings measured by EEG. This suggests that $\mathbf{A}$ effects on $\mathrm{Ca}^{2+}$ wave functions may maximize their influence on STM at frequencies consistent with synchronous EEG during STM by some mechanisms not yet determined.

\subsubsection{Coherence of Regenerative Processes}

The short summary above argues for Zeno-type long-time coherence of $\mathrm{Ca}^{2+}$ wave packets, during which they are affected by the $\mathrm{P}=\mathbf{p}+q \mathbf{A}$ interaction due to highly synchronous neural activity, and in turn their role in modifying synaptic interactions is affected. This study using qPATHTREE and qPATHINT is motivated to further calculate just how much shock the $\mathrm{Ca}^{2+}$ wave packets may endure by virtue of the regenerative process that defines it.

\section{CONCLUSION}

Algorithms originally developed for classical complex financial options, PATHTREE and PATHINT, have been generalized to complex variable spaces, resulting in a new code useful for quantum wave functions and/or quantum probability functions, qPATHTREE and qPATHINT. The essence of PATHTREE is its ability to process quite generally time-dependent and nonlinear Lagrangians, and this is also useful for qPATHTREE. The PATHTREE algorithm is surprisingly fast, robust and accurate. For another path-integral code, PATHINT, also already applied to various systems, including chaotic studies, neuroscience and finance, the time and space variables are determined independently. I.e., the ranges of the space variables are best determined by first determining the reasonable spread of the distribution at the final time epoch. For PATHTREE just one parameter, the number of epochs, determines the mesh for both time and the space variables. This typically leads to a growth of the tree, proportional to the square root of the number of epochs, much faster than the spread of the distribution, so that much of the calculation is not relevant.

Much CPU in both qPATHTREE and qPATHINT is used just calculating the distribution at all nodes. The added generalization of dealing with $\mathrm{N}$ dimensions in qPATHINT, which eventually will be added to qPATHTREE, requires a lot of overhead taking care of indices and boundaries within many for-loops. Parallel processing can at least make these codes more efficient in real-time. qPATHTREE's and qPATHINT's accuracy is best for moderate-noise systems. qPATHTREE is applied to a free particle. Calculations are give in the presence of random serial shocks to the particle. The strength of the code is seen only in the region of the initial value. Although oscillatory behavior is not reproduced, the current qPATHTREE is shown to be useful to study some aspects of serial changes to systems. qPATHINT also applied to a free particle, and it does handle oscillatory system in it present form that has a wider span than qPATHTREE over independent variables at each time slice.The current qPATHINT does handle more general systems, as it can easily span wider ranges of variables. Codes under development for qPATHTREE and qPATHINT will have sufficient spans of multiple deviations on each branch to address such oscillatory behavior. qPATHTREE can be similarly developed.The example a free quantum particle was chosen to test treatment of its oscillatory properties. However, of course this system has an infinite domain, and so calculations performed here cannot be accurate given the finite ranges used. However, a proof of principle has been demonstrated, which is poised to handle quantum options on quantum systems, in finance and in blockchains. qPATHTREE and qPATHINT can be useful in multiple disciplines, e.g., neuroscience and financial markets. Both qPATHTREE and qPATHINT will be further generalized to $\mathrm{N}$-variable parallel code in complex-variable spaces.

\section{ACKNOWLEDGMENT}

I thank the National Science Foundation's Extreme Science and Engineering Discovery Environment (XSEDE.org), for three supercomputer grants since February 2013, "Electroencephalographic field influence on calcium momentum waves", one under PHY130022 and two under TG-MCB140110. The current grant also under TG-MCB140110, “Quantum path-integral qPATHTREE and qPATHINT algorithms", runs through June 2017.

\section{REFERENCES}

[1]. L. Ingber, C. Chen, R.P. Mondescu, D. Muzzall, and M. Renedo. Probability tree algorithm for general diffusion processes. Physical Review E, 64(5):056702-056707, 2001. https://www.ingber.com/path01_pathtree.pdf.

[2]. L. Ingber. Statistical mechanics of neocortical interactions: Path-integral evolution of short-term memory. Physical Review E, 49(5B):4652-4664, 1994. https://www.ingber.com/smni94_stm.pdf. 
[3]. L. Ingber. Path-integral evolution of multivariate systems with moderate noise. Physical Review E, 51(2):1616-1619, 1995. https://www.ingber.com/path95_nonl.pdf.

[4]. L. Ingber. High-resolution path-integral development of financial options. Physica A, 283(3-4):529-558, 2000. https://www.ingber.com/markets00_highres.pdf.

[5]. L. Ingber and P.L. Nunez. Statistical mechanics of neocortical interactions: High resolution path-integral calculation of short-term memory. Physical Review E, 51(5):5074-5083, 1995. https://www.ingber.com/smni95_stm.pdf.

[6]. L. Ingber, R. Srinivasan, and P.L. Nunez. Path-integral evolution of chaos embedded in noise: Duffing neocortical analog. Mathematical Computer Modelling, 23(3):43-53, 1996. https://www.ingber.com/path96_duffing.pdf.

[7]. S. Aaronson and P. Christiano. Quantum money from hidden subspaces. Technical Report arXiv:1203.4740 [quant-ph], MIT, Cambridge, MA, 2012.

[8]. L. Accardi and A. Boukas. The quantum black-scholes equation. Technical Report arXiv:0706.1300 [q-fin.PR], U di Roma Torvergata, Rome, 2007.

[9]. B.E. Baaquie, C. Coriano, and M. Srikant. Quantum mechanics, path integrals and option pricing: Reducing the complexity of finance. Technical Report arXiv:cond-mat/0208191 [cond-mat.soft], National U Singapore, Singapore, 2002.

[10]. J. Jogenfors. Quantum bitcoin: An anonymous and distributed currency secured by the no-cloning theorem of quantum mechanics. Technical Report arXiv:1604.01383 [quant-ph], Linkoping U, Linkoping, Sweden, 2016.

[11]. K. Meyer. Extending and simulating the quantum binomial options pricing model. Technical Report Thesis, U Manitoba, Winnipeg, Canada, 2009. http://hdl.handle.net/1993/3154.

[12]. E.W. Piotrowski, M. Schroeder, and A. Zambrzycka. Quantum extension of european option pricing based on the ornstein-uhlenbeck process. Physica A, 368(1):176-182, 2005.

[13]. L. Ingber. Statistical mechanics of neocortical interactions. i. basic formulation. Physica D, 5:83-107, 1982. https://www.ingber.com/smni82_basic.pdf.

[14]. L. Ingber. Statistical mechanics of neocortical interactions. dynamics of synaptic modification. Physical Review A, 28:395-416, 1983. https://www.ingber.com/smni83_dynamics.pdf.

[15]. L. Ingber. Statistical mechanics of neocortical interactions. derivation of short-term-memory capacity. Physical Review A, 29:3346-3358, 1984. https://www.ingber.com/smni84_stm.pdf.

[16]. L. Ingber. Statistical mechanics of neocortical interactions: Stability and duration of the 7+-2 rule of short-term-memory capacity. Physical Review A, 31:1183-1186, 1985. https://www.ingber.com/smni85_stm.pdf.

[17]. F. Langouche, D. Roekaerts, and E. Tirapegui. Discretization problems of functional integrals in phase space. Physical Review D, 20:419-432, 1979.

[18]. K. Schulten. Non-equilibrium statistical mechanics. Technical Report Lecture Notes, U. Illinois, Urbana, IL, 2000.

[19]. J.C. Hull. Options, Futures, and Other Derivatives, 4th Edition. Prentice Hall, Upper Saddle River, NJ, 2000.

[20]. Y.K. Kwok. Mathematical Models of Financial Derivatives. Springer-Verlag, Singapore, 1998.

[21]. F. Black and M. Scholes. The pricing of options and corporate liabilities. The Journal of Political Economy, pages 637-659, 1973.

[22]. M.F. Wehner and W.G. Wolfer. Numerical evaluation of path-integral solutions to fokker-planck equations. I. Physical Review A, 27:2663-2670, 1983a.

[23]. M.F. Wehner and W.G. Wolfer. Numerical evaluation of path-integral solutions to fokker-planck equations. II. restricted stochastic processes. Physical Review A, 28:3003-3011, 1983 b.

[24]. M.F. Wehner and W.G. Wolfer. Numerical evaluation of path integral solutions to fokker-planck equations. III. time and functionally dependent coefficients. Physical Review A, 35:1795-1801, 1987.

[25]. J. Conway and S. Kochen. The free will theorem. Technical Report arXiv:quant-ph/0604079 [quant-ph], Princeton U, Princeton, NJ, 2006.

[26]. J. Conway and S. Kochen. The strong free will theorem. Notices of the American Mathematical Society, 56(2):226-232, 2009.

[27]. A. Pereira and F.A. Furlan. On the role of synchrony for neuron-astrocyte interactions and perceptual conscious processing. Journal of Biological Physics, 35(4):465-480, 2009.

[28]. E. Scemes and C. Giaume. Astrocyte calcium waves: What they are and what they do. Glia, 54(7):716-725, 2006. http://dx.doi.org/10.1002/glia.20374.

[29]. B. Innocenti, V. Parpura, and P.G. Haydon. Imaging extracellular waves of glutamate during calcium signaling in cultured astrocytes. Journal of Neuroscience, 20(5):1800-1808, 2000.

[30]. W.N. Ross. Understanding calcium waves and sparks in central neurons. Nature Reviews Neuroscience, 13:157-168, 2012.

[31]. L. Ingber. Calculating consciousness correlates at multiple scales of neocortical interactions. In A. Costa and E. Villalba, editors, Horizons in Neuroscience Research, pages 153-186. Nova, Hauppauge, NY, 2015. ISBN: 978-1-63482-632-7. Invited paper. https://www.ingber.com/smni15_calc_conscious.pdf.

[32]. L. Ingber. Statistical mechanics of neocortical interactions: Large-scale EEG influences on molecular processes. Journal of Theoretical Biology, 395:144-152, 2016. https://www.ingber.com/smni16_large-scale_molecular.pdf and 
http://dx.doi.org/10.1016/j.jtbi.2016.02.003.

[33]. L. Ingber, M. Pappalepore, and R.R. Stesiak. Electroencephalographic field influence on calcium momentum waves. Journal of Theoretical Biology, 343:138-153, 2014. https://www.ingber.com/smni14_eeg_ca.pdf and http://dx.doi.org/10.1016/j.jtbi.2013.11.002.

[34]. L. Ingber. Computational algorithms derived from multiple scales of neocortical processing. In A. Pereira, E. Massad, and N. Bobbitt, editors, Pointing at Boundaries: Integrating Computation and Cognition on Biological Grounds, pages 1-13. Springer, New York, 2011. Invited Paper. https://www.ingber.com/smni11_cog_comp.pdf and http://dx.doi.org/10.1007/s12559-011-9105-4.

[35]. L. Ingber. Columnar EEG magnetic influences on molecular development of short-term memory. In G. Kalivas and S.F. Petralia, editors, Short-Term Memory: New Research, pages 37-72. Nova, Hauppauge, NY, 2012. Invited Paper. https://www.ingber.com/smni11_stm_scales.pdf.

[36]. P.L. Nunez, R. Srinivasan, and L. Ingber. Theoretical and experimental electrophysiology in human neocortex: Multiscale correlates of conscious experience. In M.M. Pesenson, editor, Multiscale Analysis and Nonlinear Dynamics: From genes to the brain, pages 149-178. Wiley, New York, 2013. http://dx.doi.org/10.1002/9783527671632.ch06.

[37]. P. Facchi, D.A. Lidar, and S. Pascazio. Unification of dynamical decoupling and the quantum zeno effect. Physical Review A, 69(032314):1-6, 2004.

[38]. P. Facchi and S. Pascazio. Quantum zeno dynamics: mathematical and physical aspects. Journal of Physics A, 41(493001):1-45, 2008.

[39]. G. Giacosa and G. Pagliara. Quantum zeno effect by general measurements. Physical Review A, 052107:1-5, 2014.

[40]. W. Kozlowski, S.F. Caballero-Benitez, and I.B. Mekhov. Non-hermitian dynamics in the quantum zeno limit. Technical Report arXiv:1510.04857 [quant-ph], U Oxford, Oxford, UK, 2015.

[41]. M.M. Muller, S. Gherardini, and F. Caruso. Quantum zeno dynamics through stochastic protocols. Technical Report arXiv:1607.08871 [quant-ph], 2016.

[42]. Y.S. Patil, S. Chakram, and M. Vengalattore. Measurement-induced localization of an ultracold lattice gas. Physical Review Letters, 115(140402):1-5, 2015. http://link.aps.org/doi/10.1103/PhysRevLett.115.140402.

[43]. S.L. Wu, L.C. Wang, and X.X. Yi. Time-dependent decoherence-free subspace. Journal of Physics A, 405305:1-11, 2012.

[44]. P. Zhang, Q. Ai, Y. Li, D. Xu, and C. Sun. Dynamics of quantum zeno and anti-zeno effects in an open system. Science China Physics, Mechanics, Astronomy, 57(2):194-207, 2014. http://dx.doi.org/10.1007/s11433-013-5377-x.

[45]. S. Murakami and Y. Okada. Contributions of principal neocortical neurons to magnetoencephalography and electroencephalography signals. Journal of Physiology, 575(3):925-936, 2006.

[46]. P.L. Nunez and R. Srinivasan. Electric Fields of the Brain: The Neurophysics of EEG, 2nd Ed. Oxford University Press, London, 2006. 